\title{
Notes on Sources
}

This project presented a curatorial challenge. Only a fraction of the nearly two hundred texts that I initially considered for the volume are included here. Ultimately, the principle of selection was based on two criteria: authorship and exemplary or unique expression of anticolonial thought. Most of the texts below were written by students. Occasionally, texts written by faculty or administrators were included when they represented an important moment or conversation at the university about students or students' roles in society. Sources that reflected exemplary or unique perspectives were often chosen over manifestos that were, generally, the most common type of student-authored anticolonial text. After all, a book of only manifestos would soon become a tedious read. The sources reflect a variety of genres, including manifesto, memoir, legislative decree, interview, and poetry. In order to capture a range of understandings and deployments of anti-colonial theory, I also selected texts that pursued anti-colonial perspectives in unique ways. Given the scope of the series, documents that outlined global solidarities and affinities between Central American student movements were especially important.

The texts included in this volume came from many different archives, libraries, and microfilm collections. Below they are listed by chapter in the original Spanish with archival information in the order in which they appear in the book.

Chapter 1. GUATEMALA: 'En plena tiranía', El Estudiante, Año I, No. 13, 14 March 1920; Manuel Galich (1940), Del pánico al ataque, Guatemala: Tipografía Nacional, 149-55. Centro de Investigaciones Regionales de Mesoamérica (CIRMA); Asociación de Estudiantes Universitarios (AEU), 'Manifiesto', 26 January 1946. Collection of Arturo Taracena, Doc. No. 1709. CIRMA. EL SALVADOR: 'Discurso pronunciado por el doctor Miguel Rafael Urquia, en el acto solemne de la 
apertura de clases universitarias del corriente año lectivo, el 1o de Marzo de 1933', La Universidad, 30 September 1933; 'Discurso pronunciado por el Br Reinaldo Galindo Pohl, a nombre de la Asociación General de Estudiantes Universitarios (A.G.E.U.S.)', La Universidad, 1944. NICARAGUA: Universidad Central de Nicaragua (1941), Universidad Central de Nicaragua: memoria de su fundación, 15 de septiembre de 1941, Managua: Talleres Nacionales; David Sánchez Sánchez, 'El estudiantado como fuerza política', El Universitario, 27 December 1945. Instituto de Historia de Nicaragua y Centroamérica (IHNCA); Juan F. Gutiérrez, 'Hagamos patria', El Universitario, 1st week of March 1946. IHNCA. COSTA RICA: “ “En Costa Rica nos enorgullecemos de nuestra libertad de pensamiento, y todos los costarricenses estamos acostumbrados a discutir y criticar los actos de los hombres públicos," dicen los miembros de la liga anti-imperialista de estudiantes de derecho', La Tribuna, 29 April 1931; Manuel Mora Valverde (1940), Imperialismo: nuestra soberanía frente al Departamento del Estado, San José: Partido Comunista de Costa Rica. HONDURAS: Jorge Fidel Durón, 'Función de la universidad', Revista de la universidad, 12 December 1949; Jorge St. Siegens, 'Cooperativismo en Honduras', Revista de la universidad, 11 August 1950.

Chapter 2. GUATEMALA: Comité de Estudiantes Universitarios Anticomunistas Guatemaltecos en Exilio (CEUAGE), 'De pie frente a la dictadura roja en Guatemala', Boletín de CEUAGE, Vol. 1, No. 1, June 1953. Hemeroteca Nacional (HN); Comité de Estudiantes Anticomunistas Universitarios (1954), Plan de Tegucigalpa, Tegucigalpa: CEUA. Archivo Vertical, No. D-1489. CIRMA; 'AEU contra discriminación', Informador Estudiantil, January-February 1956. General Document Collection, No. 4152. CIRMA; Editorial, El Estudiante, Época II, No. 3, 19 September 1957. Collection of Arturo Taracena, No. 229. CIRMA; Asociación de Estudiantes 'El Derecho', 'La Asociación de Estudiantes el Derecho impugnando el decreto 1215 del congreso de la república, declara a Carlos Castillo Armas, traidor de la patria', 17 January 1958. Collection of Arturo Taracena, No. 106. CIRMA. EL SALVADOR: AGEUS, 'Manifiesto', Opinión Estudiantil, 16 October 1956. IHNCA; AGEUS, 'Solidaridad con los exiliados de Nicaragua', Opinión Estudiantil, 16 October 1956. IHNCA; 'Comunicación', Opinión Estudiantil, 16 October 1956. IHNCA. NICARAGUA: 'Aquí están los asesinos y sus víctimas', El Universitario, 1960. IHNCA; 'COSEC recibe cablegrama de SOMOZA', El Universitario, 1960. IHNCA. HONDURAS: UNAH (1957), 'Asamblea Nacional Constituyente de 1957', Estudio Histórico-Jurídico sobre la autonomía 
de la Universidad Nacional Autónoma de Honduras, Tegucigalpa: UNAH. 'Ley Orgánica de la Universidad de Honduras', Decreto Legislativo No. 170, 30 April 1958.

Chapter 3. NICARAGUA: 'Cuba y Latinoamérica sí! Yankis no!', El Universitario, September 1960. IHNCA; Centro Universitario de la Universidad Nacional (CUUN) (1971), Imperialismo, Managua: Editorial José Martí. Doc. No. 71. Instituto de Historia de Nicaragua (IHN). Guatemala: Jaime F. Pineda S., 'La participación del estudiante universitario en la vida nacional', Tribuna Económica (Órgano de la Asociación de Estudiantes de Ciencias Económicas), Año III, No. 25, 31 May 1962. Figueroa Ibarra Collection, No. 197. CIRMA; 'Comunicado de la Rectoría de la Universidad de San Carlos sobre el préstamo que el gobierno de la república ha contratado con el BID con destino a instituciones privadas de enseñanza superior . . ,', 7 June 1974. CIRMA; 'Fondo Monetario Internacional, tentáculo del capitalismo', 7 Días en la USAC, 3-9 March 1980. Figueroa Ibarra Collection, No. 172. CIRMA; 'Carta de un ladrón a sus vecinos'. Inforpress Centroamericana Archive, No. 478. CIRMA. COSTA RICA: FEUCR, 'Que la historia señale nuestra posición', El Universitario, May 1970. North American Congress on Latin America (NACLA); 'Porque participé en los actos del 24', El Universitario, May 1970. NACLA; Editorial, El Universitario, November 1970. NACLA. HONDURAS: 'La FEUH y la AGEUS se pronuncian', Boletín del Instituto de Investigaciones Económicas y Sociales, No. 24-5 (January and February 1974). NACLA; 'Los asesinos de la CIA', Presencia Universitaria, January 1976. NACLA. EL SALVADOR: Consejo Superior Universitaria (1964), Libertad y cultura en torno al debate universitario, San Salvador: Universidad Nacional; Asociación General de Estudiantes Universitarios Salvadoreños, 'En torno al problema agrario', El Universitario, 15 November 1979. NACLA; Asociación General de Estudiantes Universitarios Salvadoreños (1981), Salud en El Salvador, otra razón para el combate popular, San Salvador: Universidad de El Salvador, Instituto de la Historia de Centroamérica (IHCA).

Chapter 4. NICARAGUA: Carlos Fonseca (2006), 'Mensaje a los estudiantes revolucionarios', Obra fundamental, Managua: Aldilá Editor. IHNCA. GUATEMALA: Editorial, No nos tientes, 1966. Satirical Publications Collection, No. 44. CIRMA; Juventud Patriótica del Trabajo (JPT), 'A quién servirá la guerra en Belice?' Juventud, JulyAugust 1977. Archivo Inforpress Centroamericana, No. 913; CIRMA; Asociación de Estudiantes Universitarias (1977), 'El Movimiento Estudiantil Guatemalteco en la lucha por el respeto a los derechos 
humanos y democráticos y por contener la escalada de terror fascista en Guatemala', Jornadas de agosto, Guatemala: USAC Facultad de Ciencias Económicas. Collection of Robert Trudeau, No. 498; CIRMA; Oliverio Castañeda de León (1978), 'Discurso en la toma de posesión del Secretariado General de AEU, May 22, 1978', in Rebeca Alonzo Martínez (ed.), Oliverio vive!, Guatemala: Ediciones CEUR. EL SALVADOR: 'Pronunciamiento del Consejo Superior Universitario de la Universidad de El Salvador sobre la situación nacional', El Universitario, 18 December 1979. NACLA; 'Comunicado', Opinión estudiantil, July 1980. NACLA; ‘Guatemala: En la dura lucha por su libertad. . .', Opinión estudiantil, July 1980. NACLA; José María Cuellar (1979), '1932' and 'Guerras en mi país', Poemas, San Salvador: Editorial Universitaria de El Salvador; 'Poesía de una heroica mujer de nuestro pueblo', Opinión estudiantil, June 1981. NACLA. HONDURAS: 'Honduras-Nicaragua: se constituye comité de solidaridad', Presencia Universitaria, January 1976. NACLA; Armando Valladares (1978), 'Alcances y significado de nuestra autonomía universitaria', in Universidad y autonomía: un encuentro del presente, Tegucigalpa: Editorial Universitaria UNAH.

Chapter 5. COSTA RICA: R. Morua, 'Los estudiantes decimos: "Queremos construir nuestro mañana”, El Universitario, May 1970. NACLA. HONDURAS: Federación de Estudiantes Universitarios de Honduras (FEUH), 'Declaración Pública', Presencia Universitaria, January 1976. NACLA; Enrique Astorga Lira, 'Modelos marginales de reforma agraria en América latina: el caso de Honduras', Alcaraván, No. 3, April 1980; Editorial, El tornillo sin fin: el órgano viril de los estudiantes universitarias de Honduras, 1981. NACLA. GUATEMALA: Boletín No. 2 de la Huelga de Dolores, 1980. Robert Trudeau Collection, No. 482. CIRMA; Saúl Osorio Paz, 'Carta abierta del Rector Saúl Osorio Paz al Consejo Superior Universitario', 1980. Robert Trudeau Collection, No. 486. CIRMA. EL SALVADOR: 'Los estudiantes salvadoreños ante la intervención imperialista en El Salvador', Opinión Estudiantil, July 1981. NACLA; AGEUS, 'Augustín Farabundo Martí, ejemplo de la lucha antiimperialista y de la guerra popular de liberación', Opinión Estudiantil, February 1982. IHNCA. NICARAGUA: Daisy Zamora and Margarita Randall, trans. 'Commander Two', 'Report of the Demonstration in Front of the US Embassy Protesting the Pino Grande Manœuvres', and 'Song of Hope', in Zamora and Randall (2002), Clean Slate, 44-5, 118-19, 42-3. Permission for the inclusion of these poems was generously granted by the author and Northwestern University Press as rights holder; Jaime Wheelock Román (1983), 'La 
xviii Anti-Colonial Texts from Central American Student Movements

universidad por la independencia económica: la universidad beligerante' in Hacia la independencia nacional por la revolución: la universidad beligerante, Managua: UNAN. IHNCA. 
For the students - past, present, and future - of course

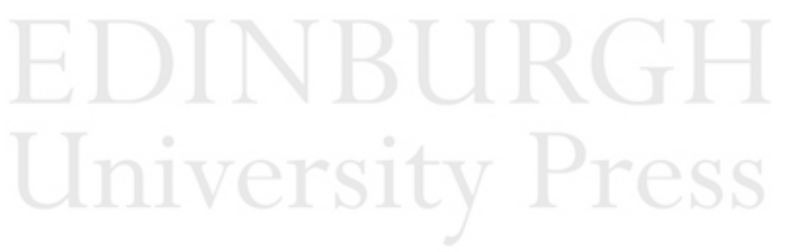




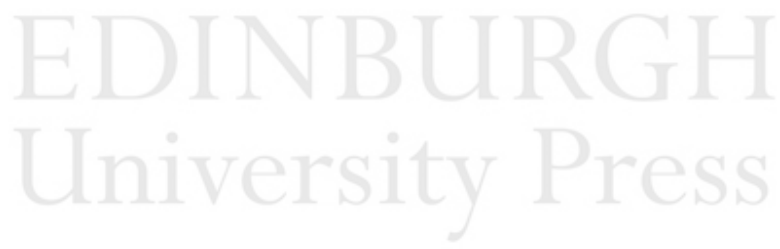

Not for distribution or resale. For personal use only. 